\title{
UNION CONDITIONS IN NOETHER LATTICES
}

\author{
JOHNNY A. JOHNSON ${ }^{1}$
}

\begin{abstract}
In this paper we introduce the strong union condition on elements and the strong union condition on primes in Noether lattices and prove some structural properties of Noether lattices which satisfy these conditions.
\end{abstract}

1. Introduction. In two recent joint papers E. W. Johnson and J. P. Lediaev introduced the concepts of the weak union condition [2] and the union condition on primes [3] in their studies of the structure of Noether lattices. In this paper we investigate the structure of Noether lattices which satisfy two closely related conditions: the strong union condition on elements, and the strong union condition on primes. In addition, for each element $A$ of a Noether lattice $L$, we introduce an element associated with $A$ which we denote by $\mathscr{A}(A)$. In conjunction with these concepts, we are able to prove the following result (Theorem 3.1).

Let $L$ be a Noether lattice. Then the following four properties are equivalent.

(1.1) $L$ satisfies the strong union condition on elements.

(1.2) $L$ satisfies the strong union condition on primes.

(1.3) For each prime element $P$ of $L$, there exists a principal element $E$ of $L$ such that $P=\mathscr{A}(E)$.

(1.4) For each element $A$ of $L$, there exists a principal element $E$ of $L$ such that $\mathscr{A}(A)=\mathscr{A}(E)$.

2. Preliminary remarks. In this section we shall define the necessary concepts and present a preliminary result. The reader is referred to [1]-[6] for terminology used in the remainder of this paper which is not defined here. Most of the basic terminology can be found in [1].

A Noether lattice $L$ is said to satisfy the strong union condition on elements if, given a collection $\left\{P_{\alpha}: \alpha \in S\right\}$ of primes of $L$ and an element $A$ of $L$ such that $A P_{\alpha}$, for each $\alpha$ in $S$, there exists a principal element $E$ of $L$ such that $E \leqq A$ and $E \$ P_{\alpha}$, for each $\alpha$ in $S$.

Received by the editors October 27, 1970.

AMS 1970 subject classifications. Primary 06A20; Secondary 06A50.

Key words and phrases. Noether lattice, principal element, ascending chain condition.

1 This research was partially supported by the Jet Propulsion Laboratory, California Institute of Technology, under Contract No. NAS7-100, sponsored by the National Aeronautics and Space Administration.

(c) American Mathematical Society 1972 
A Noether lattice $L$ is said to satisfy the strong union condition on primes if, given a collection $\left\{P_{\alpha}: \alpha \in S\right\}$ of primes of $L$ and a prime element $P$ of $L$ such that $P P_{\alpha}$, for each $\alpha$ in $S$, there exists a principal element $E$ of $L$ such that $E \leqq P$ and $E \$ P_{\alpha}$, for each $\alpha$ in $S$.

Let $L$ be a Noether lattice. For each element $A$ of $L$, let $\mathscr{A}(A)$ denote the join of all elements $X$ of $L$ such that $X^{k} \leqq A$, for some nonnegative integer $k$. Note that $A \leqq \mathscr{A}(A)$ and that, since $L$ satisfies the ascending chain condition, if $B \leqq \mathscr{A}(A)$, then there exists a nonnegative integer $k$ such that $B^{k} \leqq A$.

We will need the following preliminary result in the proof of our main theorem.

Lemma 2.1. Let $L$ be a Noether lattice, and let $A, B$ be elements of $L$. Then

(2.1) If $A \leqq B$, then $\mathscr{A}(A) \leqq \mathscr{A}(B)$.

(2.2) If $A$ is prime, then $\mathscr{A}(A)=A$.

(2.3) $\mathscr{A}(A) \wedge \mathscr{A}(B)=\mathscr{A}(A \wedge B)=\mathscr{A}(A B)$.

Proof. The proof of (2.1) and (2.2) is straightforward and will be omitted. To prove (2.3), notice that $A B \leqq A \wedge B \leqq A$, so that by (2.1) it follows that

$$
\mathscr{A}(A B) \leqq \mathscr{A}(A \wedge B) \leqq \mathscr{A}(A) \wedge \mathscr{A}(B) .
$$

Now since $L$ satisfies the ascending chain condition, there exist integers $m$ and $n$ such that

(2.4) $(\mathscr{A}(A) \wedge \mathscr{A}(B))^{m} \leqq \mathscr{A}(A)^{m} \leqq A$ and

(2.5) $(\mathscr{A}(A) \wedge \mathscr{A}(B))^{n} \leqq \mathscr{A}(B)^{n} \leqq B$.

By combining (2.4) and (2.5) we have

so that

$$
(\mathscr{A}(A) \wedge \mathscr{A}(B))^{m+n} \leqq A B,
$$

$$
\mathscr{A}(A) \wedge \mathscr{A}(B) \leqq \mathscr{A}(A B)
$$

which completes the proof.

3. The main result. In this section we shall prove our main theorem which shows a structural property of Noether lattices which satisfy the union conditions.

Let $L$ be a Noether lattice. It is immediate from the definitions that if $L$ satisfies the strong union condition on elements, then $L$ satisfies the strong union condition on primes.

Assume for a moment that there exists a prime $P$ in $L$ such that $P \neq \mathscr{A}(E)$, for every principal element $E$ of $L$ with $E \leqq P$. For each principal element $E$ of $L$ with $E \leqq P$, we shall now construct a prime element $P_{E}$ of $L$ such that $E \leqq P_{E}$ and $P$ 
the strong union condition on primes. Let $E$ be a principal element of $L$. Let $E=Q_{1} \wedge \cdots \wedge Q_{m}$ be a normal decomposition of $E$. Then, by Lemma 2.1 , we have

$$
\begin{aligned}
\mathscr{A}(E) & =\mathscr{A}\left(Q_{1} \wedge \cdots \wedge Q_{m}\right) \\
& =P_{1} \wedge \cdots \wedge P_{m}=P_{i_{1}} \wedge \cdots \wedge P_{i_{n}},
\end{aligned}
$$

where the $P_{i_{j}}$ are the isolated prime components of $E$. Since $P_{i_{1}} \cdots P_{i_{n}} \leqq$ $P_{i_{1}} \wedge \cdots \wedge P_{i_{n}} \leqq P$, and $P$ is prime, there exists an integer $k$ such that $P_{i_{k}} \leqq P$. We may assume without loss of generality that $P_{i_{1}} \leqq P$. If $n=1$, set $P_{E}=P_{i_{1}}$. Then $E \leqq P_{E}$ and $P \$ P_{E}$ since $P \neq \mathscr{A}(E)$. If $n \geqq 2$, then it must be the case that $P \leqq P_{i_{2}}$. For, if $P \leqq P_{i_{2}}$, then $P_{i_{1}} \leqq P \leqq P_{i_{2}}$, which contradicts the fact that $P_{i_{1}}$ and $P_{i_{2}}$ are isolated prime components of $E$. Thus, in this case set $P_{E}=P_{i_{2}}$. So now $E \leqq P_{E}$ and $P \leqq P_{E}$.

Let us now assume that, for each prime $P$ in $L$, there exists a principal element $E \leqq P$ in $L$ such that $P=\mathscr{A}(E)$. Let $A$ be an element of $L$. We will show that there exists a principal element $H$ of $L$ such that $H \leqq A$ and $\mathscr{A}(A)=\mathscr{A}(H)$. Let $A=Q_{1} \wedge \cdots \wedge Q_{m}$ be a normal decomposition of $A$. By Lemma 2.1 we obtain

$$
\begin{aligned}
\mathscr{A}(A) & =\mathscr{A}\left(Q_{1} \wedge \cdots \wedge Q_{m}\right)=\mathscr{A}\left(Q_{1}\right) \wedge \cdots \wedge \mathscr{A}\left(Q_{m}\right) \\
& =P_{1} \wedge \cdots \wedge P_{m}=\mathscr{A}\left(E_{1}\right) \wedge \cdots \wedge \mathscr{A}\left(E_{m}\right) \\
& =\mathscr{A}\left(E_{1} \wedge \cdots \wedge E_{m}\right)=\mathscr{A}\left(E_{1} \cdots E_{m}\right),
\end{aligned}
$$

where the $P_{i}$ are the primes associated with the $Q_{i}$ and the $E_{i}$ are principal elements of $L$. Thus $E_{1} \cdots E_{m} \leqq \mathscr{A}(A)$. Choose an integer $k$ such that $\left(E_{1} \cdots E_{m}\right)^{k} \leqq \mathscr{A}(A)^{k} \leqq A$. Set $\left(E_{1} \cdots E_{m}\right)^{k}=H$. Notice that $H$ is principal since the product of principal elements in a Noether lattice is principal [1, Corollary 3.3]. We now have, again by Lemma 2.1, that

$$
\mathscr{A}(A)=\mathscr{A}\left(E_{1} \cdots E_{m}\right)=\mathscr{A}\left(\left(E_{1} \cdots E_{m}\right)^{k}\right)=\mathscr{A}(H)
$$

where $H$ is principal and $H \leqq A$.

Now, suppose that for each element $A$ of $L$ there exists a principal element $E$ of $L$ such that $\mathscr{A}(A)=\mathscr{A}(E)$. We shall now see that $L$ satisfies the strong union condition on elements. Assume $A$ is an element of $L$ and that $A \Varangle P_{\alpha}$, for all $\alpha$ in $S$. Let $E$ be a principal element of $L$ such that $\mathscr{A}(E)=\mathscr{A}(A)$. Then $\mathscr{A}(E) \$ P_{\alpha}$, for all $\alpha$ in $S$, so $E \$ P_{\alpha}$, for all $\alpha$ in $S$.

We have now established the following result.

THEOREM 3.1. Let $L$ be a Noether lattice. Then the following four properties are equivalent.

(3.1) L satisfies the strong union condition on elements.

(3.2) L satisfies the strong union condition on primes. 
(3.3) For each prime element $P$ of $L$, there exists a principal element $E$ of $L$ such that $P=\mathscr{A}(E)$.

(3.4) For each element $A$ of $L$, there exists a principal element $E$ of $L$ such that $\mathscr{A}(A)=\mathscr{A}(E)$.

The altitude of a Noether lattice $L$ is defined to be the supremum of the ranks of the prime elements of $L$. We will require the following proposition to obtain our final result.

Proposition 3.2. Let $L$ be a Noether lattice. If for each maximal element $M$ of $L$ there exists a principal element $E$ of $L$ such that $M=\mathscr{A}(E)$, then the altitude of $L$ is at most one.

Proof. Let $M$ be a maximal element of $L$ and let $E$ be a principal element of $L$ such that $\mathscr{A}(E)=M$. Since maximal elements in a Noether lattice are prime [1], it follows that $M$ is a minimal prime of $E$, so that by the Principal Element Theorem [1, Theorem 6.4] the rank of $M$ is at most one. Thus, since this is true for every maximal element of $L$, the altitude of $L$ is at most one. Q.E.D.

By combining Theorem 3.1 and Proposition 3.2 we obtain the following theorem.

THEOREM 3.3. Let $L$ be a Noether lattice. If $L$ satisfies the strong union condition on primes, then the altitude of $L$ is at most one.

\section{REFERENCES}

1. R. P. Dilworth, Abstract commutative ideal theory, Pacific J. Math. 12 (1962), 481-498. MR 26 \#1333.

2. E. W. Johnson and J. P. Lediaev, Representable distributive Noether lattices, Pacific J. Math. 28 (1969), 561-564. MR 41 \#118.

3. _- Structure of Noether lattices with join-principal maximal elements, Pacific J. Math. 37 (1971), 101-108.

4. E. W. Johnson and J. A. Johnson, M-primary elements of a local Noether lattice, Canad. J. Math. 22 (1970), 327-331. MR 41 \#1599.

5. J. A. Johnson, Quotients in Noetherian lattice modules, Proc. Amer. Math. Soc., 28 (1971), 71-74.

6. - Subspaces and altitudes in Noetherian lattice modules, Fund. Math. 66 (1970), 375-379.

California Institute of Technology, Pasadena, California 91109

Department of Mathematics, University of Houston, Houston, Texas 77004 